\title{
TELEHEALTH: OVERCOMING BARRIERS FOR RURAL HEALTHCARE
}

\author{
Sian Sunckell
}

\section{INTRODUCTION}

Rural populations face many constraints when trying to access health services in New Zealand. There is a prominent divide between rural and urban, and what services are easily accessible and available (Ministry of Health, 2004). As technology develops, it opens doors for health professionals to work with people, communities and colleagues in new ways. Technology has provided a way to combat many of the major barriers that rural populations face in relation to health services. Telehealth allows for increased collaboration with health consumers, their primary practices and specialists, as well as allowing for inter professional collaboration between rural and urban health professionals. This article will review how telehealth has been implanted in rural contexts so far, the effectiveness of the services provided, and the opportunities for further development.

Telehealth is the use of information and communication technology (ICT) to provide healthcare from a different location. The name originates from the Greek word 'tele', which means distant (Lucas, Day, \& Honey, 2016). Telehealth uses technology including telephone, internet, and video calls to provide services to clients that they would otherwise not be able to access. The use of telehealth has been slowly increasing across many clinical areas. It has overcome barriers for rural health consumers although it still holds some barriers of its own in order to be used effectively (Sanderson, 2018).

\section{BACKGROUND}

In New Zealand, 25 percent of the population live in small towns or rural areas (Ministry of Health, 2019). There is not one definition of what a rural area is. However, they are usually defined as small populations that are not included within urban boundaries. Rural areas are often geographically isolated and removed from general services (Statistics New Zealand, n.d.).

In 2004, the Ministry of Health found that rural primary healthcare providers had high numbers of patients from low socio-economic areas, patients with community service cards as well as a high number of Māori compared to non-rural areas (Ministry of Health, 2004). Migration between rural and urban areas has changed the dynamics of rural areas and diversified these populations (Statistics New Zealand, n.d.). This diverse range of people means that health providers are having to deal with an array of different health needs. Therefore, rural populations still require specialist services that are not always accessible to them.

Living in a geographically isolated rural area brings many barriers to everyday life as well as when trying to access appropriate health care. A small population over a large area means there are less services available (Kerr \& Norris, 2004). The services that are available tend to be generic with limited specialist options. Therefore rural people are required to travel further to access appropriate care (Kerr \& Norris, 2004; Statistics New Zealand, n.d.). 
Having to travel further to access appropriate care means an increase in travel time and travel costs. An example of this is that diabetic children living in rural Central Otago were having to travel six to eight hours return to go to a half hour specialist appointment in Dunedin in order to access the care they needed (Ministry of Health, 2016a). Telehealth was implemented and used effectively to allow specialists in Dunedin to consult and collaborate with clients from a distance.

The New Zealand Health Strategy identifies telehealth as a resource worth developing. The Strategy aims to strengthen telehealth in New Zealand and increase its availability to enable people to stay closer to home for longer (Ministry of Health, 2016b). When the Strategy was released in 2016, one of the goals was to have a wide range of telehealth services available throughout the country by 2020 (Ministry of Health, 2016a). It also highlights the need to diversify our health services to remove health inequities among New Zealanders (Ministry of health, 2016a). This comes from the public's increased expectation that they will be provided with equitable care regardless of location and circumstance (Lucas et al., 2016; Wright \& Honey, 2016).

\section{DISCUSSION}

The positive impacts that telehealth has on a range of clinical setting are important to recognise. Telehealth has the ability to connect people in many different ways and forms, and allows for greater collaboration between patient, family, health professionals and support staff where it may not have been possible before. Telehealth has also shown that it can empower patients to take an active role in their own health which is essential for keeping people well for longer. This will reduce the pressure on health professionals and lead to a healthier community. The Ministry of Health (2016a) recognises that telehealth has the ability to empower people thus leading to greater, more informed choices around their health.

It is evident from this literature that when used appropriately, telehealth effectively facilitates collaboration between health providers and consumers. It has proven to promote and encourage health consumers to learn and better manage their own health by allowing them to feel safe, reassured and confident while staying connected to health providers at a distance. This in turn allows for patients to stay closer to home for longer, a key point in the Health Strategy (Ministry of Health, 2016a).

The most frequently reoccurring theme emerging from the literature was the ability telehealth has to support medical staff in remote areas. Wright and Honey (2016), and O'Connor (2013a) both found that health professionals felt supported when using telehealth to collaborate with other health professionals. Telehealth has proven advantageous as it has the ability to support rural staff and lessen the professional isolation that comes with remote health services. Frequent interactions with specialist services gives confidence to rural health professionals as they feel they have the support and assistance from others with a wide range of knowledge (O'Connor, 20 I3a). This therefore helping the retention of medical staff in remote, isolated areas (Banbury, Roots, \& Nancarrow, 2014). This is important as rural areas struggle to obtain and retain medical staff. Telehealth has the potential to be adapted and developed to facilitate further collaboration with and between remote and urban areas. It could be an excellent resource to consider when looking into the retention of rural staff.

A nurse from Ashburton Hospital talks about how telehealth is used to collaborate with specialists at Christchurch hospital. Specialists are able to consult with the patient via video link, with the rural clinic nurse present too (O'Connor, 20 I3a). The rural nurse being present during the interaction gives the opportunity for them to listen to the advice and advocate for their patient when necessary. With a therapeutic nurse-patient relationship already formed, it can also be helpful if the specialist needs to give the patient bad news. O'Connor (2013a) found that the nurse present was able to support and reassure the patient and family and help them come to terms with the information they have been presented with. The three way telehealth collaboration with patient, nurse and specialist in this example worked well as it was appropriate and effective in supporting the needs of clients. This is in line with the New Zealand Health Strategy where they recognise the ability for telehealth to promote a patient focused approach to health (Ministry of Health, 2016a). 
This example of using telehealth to collaborate with specialists also meets the Nursing Council of New Zealand Competencies for registered nurses. Competency 4.1 of the Competencies for registered nurses is "collaborates and participates with colleagues and members of the healthcare team to facilitate and coordinate care" (Nursing Council of New Zealand, 2007, p.29). Telehealth was used as a resource to connect the two teams in order to collaborate and plan the ongoing care of the patient.

Telehealth also proved advantageous when used specifically with Māori populations in rural areas. A trial of telehealth was carried out in a Māori facilitated health centre in rural Gisborne where 88 percent of the trial participants were Māori (Telehealth Cutting Rural Health Barriers, 2012). Telemonitoring equipment was used by the participants and the data was sent directly to the health centre's nurse. The system allowed for secure, question and answer, online chat of which the clinic nurse was able to correspond in te reo Māori (Telehealth Cutting Rural Health Barriers, 20I2). This is an important feature as it allows the nurse to practice in a way that is deemed culturally safe by the client.

Participants in this trial were also seen to have developed a greater health literacy over the course of the trial. Participants noted that they were more interested in the numbers being displayed when taking their vital signs and learnt about what these measures meant for their health (Telehealth Cutting Rural Health Barriers, 20I2). Collaboration between the health centre and participants not only produced better health outcomes for the clients but also their families. A flow on effect from this trial was evident as families became more involved. This led to widespread family interaction and education with whole households learning how to take their vital signs and how these measures related to their own health (Telehealth Cutting Rural Health Barriers, 20I2). For participants, simply having access to their own personal health information and some education around this was enough to encourage lifestyle changes. Families started to exercise more and eat healthier in order to see a positive change in their vital signs.

Devitt (20I8) and Venter, Burns, Hefford and Ehrenberg (2012) also discovered improved health literacy among patients as a product of collaboration using telehealth. Devitt (2018) implemented telehealth in an aged care setting by remotely monitoring their vital signs and being able to provide timely access to health coaching. One patient spoke about having a better understanding of his multiple chronic conditions. This resulted in the patient managing his own health for longer, a reduction in anxiety as well as giving him an increase in self-confidence (Devitt, 2018). This led to an increase in social interaction and subsequently fewer hospital presentations. Venter et al. (2012) also looked at remote monitoring of patients with chronic conditions. They had similar results from their research including better patient understanding of medical conditions, treatment and medications. They also reported increased family engagement and the ability to provide reassurance to both patient and family around the health of their loved one (Venter et al., 20I2).

Although there are many positive aspects to telehealth, there are still some challenges to overcome when implementing the technology into rural areas. The most frequently acknowledged barrier identified in the literature was the need to have up-to-date, reliable equipment, with a suitable reliable internet connection. Lucas et al. (2016), found the unpredictable weather conditions on the West Coast were a barrier to successfully using telehealth services. With an abundance of rain and wind, power and internet services would often fail, meaning the tele-consulting options implemented were no longer able to be used. This meant staff were reluctant to use the equipment as it was not always reliable. O'Connor, (2013a) discovered that urban areas usually had state of the art equipment that was more up to date and fit for purpose than the rural areas. This lead to disparities in the functioning ability of equipment and meant poor visual and sound quality to the rural users. With limited equipment, $\mathrm{O}^{\prime}$ Connor (20 I3b) also found that nurses spent a lot of time finding and booking equipment and getting the correct consultant on the other end of the technology. This became very time consuming and discouraged staff from using telehealth to collaborate with others in this way. 
O'Connor, (2013b) found that trying to implement telehealth was not easy and proved time consuming. Doctors using telehealth in a rural emergency department spoke about it being a distraction. When other staff were using the telehealth equipment, it was loud and hard to get away from, as they work in a small area (Lucas et al., 2016). This also led to a privacy issue. In an area where everyone can hear and see what is going on, patient privacy is at risk. The solution that was implemented into the emergency department was to move the telehealth equipment into a separate room where the treating health practitioner and patient could all be in together (Lucas et al., 2016). This increased the amount of time taken for a patient consultation as it required more time to organise getting the patient into the correct room, with the correct staff and working technology. Lucas et al. (2016), drew the conclusion that although telehealth has its benefits, it was challenging to implement into an unpredictable and high pace environment like the emergency department. Although the logistics of telehealth caused problematic in this setting, the ability to collaborate and work with other health professionals from a distance was still seen as beneficial.

Davis, Hopkins, and Abrahams (2012), an Australian group using telehealth to provide speech language therapy to children, identified the need for face to face consultations alongside their telehealth services. Telehealth technology is a crucial aspect of their service because without it, many children would not have any access to regular speech language therapy unless they could afford the cost and time to travel. The feedback from their clients was that the face to face sessions were invaluable and supplemented the telehealth modules well. This shows that although telehealth services have the ability to do what is usually done face to face, it does not fully replace it. Face to face sessions are still important as they provide contact that you do not through telehealth.

Lack of face to face interaction with clients and the possibility of no longer having the same need for staff were other concerns identified throughout the literature. Wright and Honey (2016) discovered that nurses worried about the ability to form therapeutic relationships when interacting through telehealth with a patient. However, they soon found out that nurses were able to adapt to the new technology. They were still able to form the important relationships required to work in partnership with their patients (Wright \& Honey, 2016).

\section{RECOMMENDATIONS}

It is evident from the literature above that telehealth offers a wide array of possibilities to enhance the way rural healthcare is provided. The first recommendation recognises that the support telehealth offers rural health professionals by connecting them with consultants and other specialist services is invaluable (Banbury et al., 20I4; Lucas et al., 2016; Wright \& Honey, 2016). However, this could be expanded further to increase interprofessional collaboration and aid in developing rural health professionals continued education (O'Connor, 2013a).

Telehealth could be used to enable rural health professionals to join in with grand rounds, where patient cases are presented for the education of others. By having a telehealth link, rural health teams could too be included in this educational tool and collaborate with others. Telehealth could also be used to connect rural staff with inservice trainings. These frequent trainings are important as they help with continued education and development. Not only could rural areas connect and collaborate with the urban areas but also other areas in similar situations. Increased opportunities for rural health professionals to collaborate with others, continue their education, and more professional development, will help them fell more supported. These opportunities for rural health professionals could aid in the retention of staff benefiting rural and remote communities greatly.

Lucas et al. (2016), recognised poor quality equipment and lack of reliable internet services as a challenge when trying to implement telehealth it into a rural emergency department. The second recommendation is to invest funding into technology and internet services to help combat these barriers. By increasing funding for technology and internet services, we can help to bridge the rural-urban divide. Up-to-date technology with good internet service will provide clear, quality visuals and audio for the users. This will, in turn, allow for greater communication 
and assessment capabilities when collaborating with patients and health providers. Lucas et al. (2016), recognised that although the application in this environment was not favourable, telehealth still had room for development and in time they could see it working. Once telehealth can be used reliable in a clinical setting, it will increase the health professionals' confidence in the system.

These recommendations assist in working towards more equitable healthcare for rural New Zealanders. Increased professional development means the health providers in rural areas will have a higher level of skills they can use in their communities, thus reducing the need for rural people to travel further for specialist care. When rural providers are not able to offer the appropriate care needed for a patient, telehealth has the ability to connect and collaborate to overcome this. Telehealth provides a way to help bridge the current health inequities faced when living rurally (Lucas et al., 2016).

In 2020 with the global Covid-19 pandemic, there has been more reliance on telehealth services than ever before. This has increased awareness around the ability to provide health services from a distance as well as highlighting current challenges in its implementation. Telehealth in New Zealand has had to evolve and adapt to overcome new challenges faced in relation to Covid-19 (NZ Telehealth Resource Centre, 2020). Telehealth has provided a way to continue with social distancing and limit the amount of close contacts with health professionals and the public (Monaghesh \& Hajizadeh, 2020). Limiting contacts leads to decreased transmission of the disease and helps slow the spread, thus keeping communities and health professionals safer. Telehealth is proving advantageous in helping stop the spread of disease and is being recommended as a tool to utilise to carry out safer health care during the global pandemic (Monaghesh \& Hajizadeh, 2020).

\section{CONCLUSION}

Telehealth has been carried out in a variety of different clinical settings. It is important to remember that there are still challenges with successfully implementing telehealth into all areas but with further trials and development, these can be minimised. Telehealth has successfully overcome many of the barriers rural communities face when trying to access appropriate healthcare. By connecting the patient, primary health services and specialists, all parties are able to collaborate effectively, therefore providing specialist services closer to home, decreasing the time and cost taken to get to appointments as well as empowering patients to manage their own health. Connecting and collaborating via telehealth has not only had a positive impact on patients but also on their families with everyone getting involved. Telehealth is a tool that can be used to support our rural health professionals and reduce professional isolation. The opportunities telehealth holds are vast, and with the ever increasing advances in technology, it is a topic that is worth pursing for our rural and remote communities.

Sian Sunckell is a Bachelor of Nursing student in her final year of study at Otago Polytechnic, Dunedin, New Zealand. She has been a member of St John for 14 years and is now a Volunteer Ambulance Officer on frontline ambulance. Sian grew up in rural Canterbury, with strong ties to her community and St John which contributed to her interest in rural health and emergency care.

Correspondence to: Sian Sunckell, School of Nursing, Otago Polytechnic | Te Kura Matatini ki Otago, Forth Street, Private Bag 1910, Dunedin 9054, New Zealand. Email: sian.sunckell@gmail.com 


\section{REFERENCES}

Banbury, A., Roots, A., \& Nancarrow, S. (2014). Rapid review of applications of e-health and remote monitoring for rural residents. Australian Journal of Rural Health, 22(5), 21 I-22. https://doi-org.op.idm.oclc.org/I0.1 I I I/ajr.12127

Davis, A., Hopkins, T., \& Abrahams, Y. (2012). Maximizing the impact of telepractice through a multifaceted service delivery model at The Shepherd Centre, Australia. Volta Review, II2(3), 383-39I.

Devitt, A. (2018). Improving the lives of rural residents Using nurse-led telemonitoring in community aged care. Australian Nursing \& Midwifery Journal, 25(7), 32.

Kerr, K., \& Norris, T. (2004). Telehealth in New Zealand: Current practice and future prospects. Journal of Telemedicine \& Telecare, $10,60-63$

Lucas, J. A. M., Day, K., \& Honey, M. L. L. (2016). Clinician's perceptions of telehealth for emergency care on the West Coast of New Zealand: Findings of a descriptive study. Emergency Nurse New Zealand, 6-10.

Ministry of Health. (2004). A comparison of primary health care provided by rural and non-rural general practices: The national primary medical care survey (NatMedCa): 2001/02 Report 4. Wellington: Author.

Ministry of Health. (2016a). New Zealand Health Strategy: Future direction. Wellington: Author.

Ministry of Health. (2016b). New Zealand health strategy: Roadmap of actions 2016. Wellington: Author.

Ministry of Health. (2019). Rural health. Retrieved from: https://www.health.govt.nz/our-work/populations/rural-health

Monaghesh, E., \& Hajizadeh, A. (2020). The role of telehealth during COVID-19 outbreak: A systematic review based on current evidence. BMC Public Health 20, II93. https://doi.org/I0.I |86/s I2889-020-0930 I-4

NZ Telehealth Resource Centre. (2020). COVID-19. Retrieved from: https://www.telehealth.org.nz/covid-19/

Nursing Council of New Zealand. (2007). Competencies for Registered Nurses. Retrieved from https://www.nursingcouncil.org.nz/ Public/Nursing/Standards_and_guidelines/NCNZ/nursing-section/Standards_and_guidelines_for_nurses.aspx

O'Connor, T. (2013a). Videoconferences bring specialist care closer to home. Kai Tiaki Nursing New Zealand, 19(6), I5.

O'Connor, T. (20।3b). National coordination sought for telemedicine. Kai Tiaki Nursing New Zealand, 19(6), 16.

Sanderson, A. (2018). The satisfactions of telenursing. Kai Tiaki Nursing New Zealand, 24(7), 32-33

Statistics New Zealand. (n.d.). Defining urban and rural New Zealand. Retrieved from http://archive.stats.govt.nz/browse_for_ stats/Maps_and_geography/Geographic-areas/urban-rural-profile/defining-urban-rural-nz.aspx

Telehealth Cutting Rural Health Barriers. (2012, February). Nursing Review (1173-8014), 12(10), 9.

Venter, A., Burns, R., Hefford, M., \& Ehrenberg, N. (2012). Results of a telehealth-enabled chronic care management service to support people with long-term conditions at home. Journal of Telemedicine \& Telecare, 18(3), 172-175. https://doi-org. op.idm.oclc.org//0.1258/jtt.2012.SFTII2

Wright, J., \& Honey, M. (2016). New Zealand Nurses' Experience of Tele-Consultation within secondary and tertiary services to provide care at a distance. Nursing Praxis in New Zealand, 32(2), 30-38. 